\title{
Relationship between serum hepcidin levels and cardiovascular disease in patients with maintenance hemodialysis
}

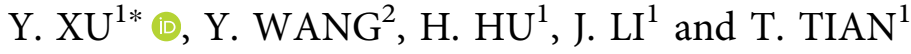 \\ ${ }^{1}$ Hemodialysis Center, Shanghai Yangsi Hospital, Pudong New Area, Shanghai, China \\ ${ }^{2}$ Hemodialysis Center, Yantai Affiliated Hospital of Binzhou Medical University, Yantai City, Shandong \\ Province, China
}

Received: March 26, 2020 - Accepted: July 06, 2020

Published online: December 18, 2020

() 2020 Akadémiai Kiadó, Budapest

\begin{abstract}
Background: To investigate the serum level of hepcidin and its relationship with cardiovascular disease (CVD) in maintenance hemodialysis (MHD) patients. Methods: Blood was obtained from 75 MHD patients before undergoing hemodialysis and 20 healthy controls. Serum hepcidin, advanced oxidation protein products (AOPP) and interleukin (IL)- 6 were measured by enzyme-linked immunosorbant assay (ELISA). Spearman correlation, and binary logistic regression linear regression analyses were used to assess the relationship between serum hepcidin and other parameters. Results: The serum level of hepcidin, AOPP and IL-6 was significantly up-regulated in MHD patients compared with the control ( $P$ $<0.05)$. Furthermore, serum hepcidin levels in patients with CVD were higher than those in patients without CVD $(P<0.05)$. In all MHD patients, serum hepcidin level was correlated positively with erythropoietin (EPO) dose per week $(\rho=0.251, P=0.030)$, EPO resistance index $(\rho=0.268, P=$ $0.020)$, ferritin $(\rho=0.814, P<0.001)$, transferin saturation (TSAT, $\rho=0.263, P=0.023)$, AOPP $(\rho=$ $0.280, P=0.049)$, high sensitive $C$ reactive protein $(\rho=0.151, P=0.006)$, IL-6 $(\rho=0.340, P=0.003)$ and left ventricular mass index (LVMI, $\rho=0.290, P=0.033$ ). Moreover, it was negatively correlated with serum pre-albumin $(\rho=-0.266, P=0.021)$, total iron-binding capacity (TIBC, $\rho=-0.458, P<$ 0.001 ), unsaturated iron-binding capacity (UIBC, $\rho=-0.473, P<0.001)$ and transferrin $(\rho=-0.487, P$ $<0.001)$. Linear regression analysis showed that ferritin $(\beta=0.708, P<0.001)$, TIBC $(\beta=-0.246, P=$ $0.032)$ and IL-6 $(\beta=0.209, P=0.041)$ were independently associated with hepcidin. Results of binary logistic regression analysis suggested that higher serum hepcidin level $(>249.2 \mathrm{ng} / \mathrm{mL})$ was positively and independently related to CVD $(\mathrm{OR}=1.32,95 \%$ CI $[1.20-9.56], P=0.043)$. Conclusions: Serum hepcidin
\end{abstract}

\footnotetext{
*Corresponding author. E-mail: yanxu20@yeah.net, haoyishengxu@163.com
} 
level is associated with CVD in MHD patients, indicating that hepcidin may be a novel biomarker and therapeutic target for CVD.

\section{KEYWORDS}

hepcidin, maintain hemodialysis, cardiovascular disease, relationship

\section{INTRODUCTION}

Cardiovascular disease (CVD) is responsible for approximately $45 \%$ deaths in patients under hemodialysis, but its pathogenesis is very complex [1]. The event incidence of CVD is high in the first few weeks after initiation of hemodialysis and is associated with systemic inflammation and endothelial dysfunction in hemodialysis patients $[2,3]$. CVD was proved to be associated with worse cognitive performance in maintain hemodialysis (MHD) patients [4]. Numerous factors including hypertension, fluid overload, systemic inflammation, lipid disorders, wound healing and coagulation, etc. contribute to CVD in this population [5]. However, it remains unclear which factor in MHD patients is related to CVD. Therefore, discovering new factors for understanding the pathophysiology of CVD and eventually finding new treatments for CVD are needed.

Hepcidin is an iron-regulatory peptide hormone, a 25-amino acid type-II acute-phase protein analogous to ferritin, which plays an important role in iron homeostasis [6,7]. The expression of the key iron regulatory hormone hepcidin is regulated by iron availability, inflammation, hormones, hypoxia, and anaemia. Hepcidin shows antimicrobial activity in human hepatic cells in case of iron overload or inflammation. The inflammatory cytokines, such as IL- $1 \beta$ and IL- 6 can induce hepcidin secretion $[8,9]$. Hepcidin is associated with iron absorption in the intestine and performs its different functions via a single biochemical mechanism: hepcidin-ferroportin interaction [10,11]. Furthermore, hepcidin stimulates degradation of ferroportin, and thus reduces iron supply to the bone marrow and decrease serum iron levels [12]. Recently, some scholars have suggested that high serum levels of hepcidin in hemodialysis patients can cause iron retention in vascular endothelial cells and plaque macrophages, and cause CVD through oxidative stress [13]. In an elderly general population, it is found that fibrinogen and $\gamma$-glutamyltransferase are directly related to hepcidin, but hepcidin is not correlated to CVD [14]. So far the relationship between serum hepcidin and CVD in MHD patients has not been studied in China. The purpose of this study is to explore the relationship between serum hepcidin level and CVD in MHD patients, and to provide new ideas for the treatment of CVD in MHD patients.

\section{MATERIALS AND METHODS}

\section{Patients}

In January 2019, a total of 75 MHD patients were recruited in the Blood Purification Center, Shanghai Yangsi Hospital and Yantai Affiliated Hospital of Binzhou Medical College, China. For 
hemodialysis, dialyzers were disposable, and the material of membrane was polysulfone with an area of $1.2-1.6 \mathrm{~m}^{2}$. The dialysis water was treated with reverse osmosis, standard bicarbonate dialysate, and the dialysate flow was $500 \mathrm{~mL} / \mathrm{min}$. The blood flow was $200-300 \mathrm{~mL} / \mathrm{min}$. Hemodialysis was taken 3 times a week, $4 \mathrm{~h}$ each session. Anticoagulation was performed with normal heparin or low molecular weight heparin. The vascular access was autogenous arteriovenous fistula or central venous catheterization. After 1 year of follow-up, CVD was defined as myocardial infarction, angina pectoris, heart failure, cerebral hemorrhage, cerebral infarction, internal fistula occlusion or vascular reconstruction including cardiac and peripheral blood vessels. Clinical data for patients including age, gender, body mass index (BMI), erythropoietin (EPO), EPO resistance index, smoking history, diabetes history, CVD history and dialysis related data were collected. 20 healthy people without obesity and smoking history were selected as the control.

Inclusion criteria: Patients who had MHD for more than 3 months; patients who were over 18 years old and in a stable condition. Exclusion criteria: Patients with severe heart failure, liver disease, severe infection, active tuberculosis, malignant tumor, active inflammatory disease taking immunosuppressant; patients with acute cardiovascular events in the past month; patients with peritoneal dialysis or kidney transplantation converted to hemodialysis; patients with recent surgery or blood transfusion history; and those who did not want to participate in this study. The study was approved by the Ethical Committee of Shanghai Yangsi Hospital (Ysyyllwj-208-11).

\section{Laboratory examination}

Hemoglobin ( $\mathrm{Hb}$ ), leukocyte count (WBC), serum creatinine (SCR), urea nitrogen (BUN), uric acid, calcium (CA), phosphorus (P), whole parathyroid hormone (iPTH), albumin, prealbumin, triglyceride (TG), total cholesterol (TCH), high density lipoprotein cholesterol (HDL-C), low density lipoprotein cholesterol (LDL-C), total iron-binding capacity (TIBC), unsaturated ironbinding capacity (UIBC), serum iron, transferrin, ferritin and high sensitivity $\mathrm{C}$ reactive protein (hsCRP) were measured by widely used methods. Cardiac troponin $\mathrm{T}$ (cTnT) and amino-terminal pro-B-natriuretic peptide (NT-proBNP) were detected by electrochemiluminescence. Transferrin saturation (TSAT) $=$ serum iron/TIBC.

Serum ferritin, advanced oxidation protein products (AOPP) and IL-6 were determined by enzyme-linked immunosorbent assay (ELISA). Briefly, blood was drawn from the peripheral vein of patients with MHD before dialysis or the healthy control on an empty stomach, in the morning. The blood was placed in a serum tube, and centrifuged at 1,000 $\mathrm{g}$ for $10 \mathrm{~min}$. The serum was collected and stored at $-70{ }^{\circ} \mathrm{C}$ for further testing. The samples were thawed at room temperature and tested by ELISA Kits (Wuhan Cusabio Company, China) according to the instructions. The measured optical density value was used to make the standard curve by Curve Expert Software, and the corresponding concentration of the sample was calculated.

\section{Measurement of carotid intima-media thickness}

After hemodialysis, carotid intima-media thickness (IMT) was measured by Philips SD800 ultrasound with a probe frequency of 7.5 MHz. Briefly, patients took a supine position, and the posterior wall of the proximal branch of the common carotid artery and $1 \mathrm{~cm}$ above the origin of 
the internal carotid artery of patients were measured. The real-time two-dimensional images of the transverse and longitudinal axes of the common carotid artery and its branches were continuously observed from the root of the neck. The end diastolic images were taken to measure the vertical distance from the inner surface of the lumen to the outer surface of the mesomembrane as the carotid IMT.

\section{Echocardiography}

All MHD patients underwent echocardiography within $2 \mathrm{~h}$ after HD by two experienced ultrasound doctors using a PHILIPS IE33 Doppler color ultrasound system with a probe frequency of 3.5 MHz. Patients lay on their left side, with a standard long axis view of the left ventricle beside the sternum. The systolic and diastolic wall thickness, the change of left ventricular diameter and left ventricular ejection fraction (LVEF) were measured by the method recommended by the American Society of Echocardiography. The left ventricular mass (LVM) was calculated by Devereux formula [12]. LVM $(\mathrm{g})=0.8 \times 1.04$ [(left ventricular end diastolic diameter + left ventricular posterior wall thickness + interventricular septum thickness) ${ }^{3}-$ left ventricular end diastolic diameter $\left.{ }^{3}\right]+0.6 . \mathrm{LVMI}=\mathrm{LVM} /$ height $^{2.7}$.

\section{Statistical analysis}

Statistical Product and Service Solutions (SPSS) 19.0 software (SPSS Inc., Chicago, IL, USA) was used for statistical analysis of the data. The continuity variables were first tested for normality. The continuous variables of normal distribution were represented by $\bar{x} \pm s$, the continuous variables of skew distribution were represented by median (interquartile spacing), and the population data were represented by composition ratio. The differences between the two groups were compared by nonparametric test, $\mathrm{t}$-test and $\chi 2$ test. Spearman correlation and linear regression analysis (natural logarithm transformation of skew distribution data) were used to analyze the correlation between hepcidin and each index. The correlation between CVD and each index was analyzed by binary logistic regression. All tests were unpaired, and $P<0.05$ was considered statistically significant. All CIs were $95 \%$.

\section{RESULTS}

\section{General information of patients with MHD}

General information of patients with MHD is shown in Table 1. A total of 75 patients undergoing hemodialysis including 40 males and 35 females were evaluated in this study. The mean age of patients was 59 years (range $49-72$ years). The mean duration on dialysis was 44 months (range 25-92 months). The body mass index (BMI) was $21.9 \pm 2.8 \mathrm{~kg} / \mathrm{m}^{2}$. The mean systolic blood pressure was $135.5 \pm 22.4 \mathrm{mmHg}$ and the mean diastolic blood pressure was 78.8 $\pm 12.0 \mathrm{mmHg}$.

During the follow-up, 26 patients were found to have developed CVD (34.7\%), including 8 cases of cerebral infarction (30.8\%), 5 cases of myocardial infarction (19.2\%), 4 cases of cerebral hemorrhage (15.4\%), 4 cases of heart failure (15.4\%), 2 cases of angina pectoris (7.7\%), 2 cases of peripheral vascular disease (7.7\%), and 1 case of internal fistula occlusion (3.8\%). 
Table 1. Univariate analysis of correlation between serum hepcidin and clinical and laboratory indexes in hemodialysis patients

\begin{tabular}{|c|c|c|c|}
\hline & $\bar{x} \pm s$ or $\mathrm{M}^{*}$ & $\rho$ & $P$ value \\
\hline Age (Year) & $59(49-72)$ & -0.109 & 0.355 \\
\hline BMI $\left(\mathrm{kg} / \mathrm{m}^{2}\right)$ & $21.9 \pm 2.8$ & -0.112 & 0.341 \\
\hline Dialysis duration (month) & $44(25-92)$ & -0.026 & 0.827 \\
\hline $\mathrm{spKt} / \mathrm{V}$ & $1.29(1.18-1.59)$ & -0.063 & 0.664 \\
\hline EPO dose (U/week) & $6,000(5,000-10,000)$ & 0.251 & 0.030 \\
\hline EPO resistance index & $1.17(0.65-1.72)$ & 0.268 & 0.020 \\
\hline Systolic blood pressure (mmHg) & $135.5 \pm 22.4$ & -0.238 & 0.052 \\
\hline Diastolic blood pressure (mmHg) & $78.8 \pm 12.0$ & -0.167 & 0.177 \\
\hline Serum albumin $(g / L)$ & $40.7 \pm 2.8$ & 0.052 & 0.660 \\
\hline Serum pre-albumin (g/L) & $0.36 \pm 0.09$ & -0.266 & 0.021 \\
\hline Scr before dialysis $(\mu \mathrm{mol} / \mathrm{L})$ & $1,006.1 \pm 231.3$ & -0.341 & 0.103 \\
\hline BUN before dialysis (mmol/L) & $27.7 \pm 6.5$ & -0.124 & 0.294 \\
\hline Serum uric acid $(\mu \mathrm{mol} / \mathrm{L})$ & $449.1 \pm 92.4$ & -0.097 & 0.461 \\
\hline $\mathrm{Ca}(\mathrm{mmol} / \mathrm{L})$ & $2.27(2.19-2.47)$ & 0.049 & 0.680 \\
\hline $\mathrm{P}(\mathrm{mmol} / \mathrm{L})$ & $2.07(1.63-2.61)$ & -0.323 & 0.105 \\
\hline $\mathrm{Ca} \times \mathrm{P}\left(\mathrm{mmol}^{2} / \mathrm{L}^{2}\right)$ & $5.467(3.52-6.76)$ & -0.224 & 0.053 \\
\hline iPTH $(\mathrm{pg} / \mathrm{ml})$ & $389.4(195.6-591.7)$ & -0.025 & 0.834 \\
\hline $25(\mathrm{OH}) \mathrm{D}_{3}(\mathrm{nmol} / \mathrm{ml})$ & $27.1(17.4-37.9)$ & 0.030 & 0.801 \\
\hline $\mathrm{Tch}(\mathrm{mmol} / \mathrm{L})$ & $4.65(4.20-5.47)$ & -0.066 & 0.584 \\
\hline $\mathrm{TG}(\mathrm{mmol} / \mathrm{L})$ & $1.66(1.39-2.15)$ & 0.147 & 0.120 \\
\hline HDL-C (mmol/L) & $1.19 \pm 0.30$ & -0.094 & 0.435 \\
\hline LDL-C (mmol/L) & $2.74 \pm 0.91$ & -0.075 & 0.534 \\
\hline$\beta_{2^{-}}$microglobulin $(\mathrm{mg} / \mathrm{L})$ & $31.0(23.2-34.8)$ & 0.205 & 0.082 \\
\hline $\mathrm{Hb}(\mathrm{g} / \mathrm{L})$ & $113.7 \pm 11.1$ & 0.028 & 0.809 \\
\hline WBC $\left(10^{9} / \mathrm{L}\right)$ & $5.94(5.39-7.06)$ & 0.036 & 0.756 \\
\hline UIBC $(\mu \mathrm{mol} / \mathrm{L})$ & $28.0(23.0-33.0)$ & -0.473 & $<0.001$ \\
\hline TIBC $(\mu \mathrm{mol} / \mathrm{L})$ & $40.0(36.0-44.3)$ & -0.458 & $<0.001$ \\
\hline Transferrin $(\mathrm{g} / \mathrm{L})$ & $1.97(1.70-2.17)$ & -0.487 & $<0.001$ \\
\hline Ferritin $(\mathrm{ng} / \mathrm{ml})$ & $164.7(98.2-341.7)$ & 0.814 & $<0.001$ \\
\hline Serum iron $(\mu \mathrm{mol} / \mathrm{L})$ & $11.2(9.1-16.6)$ & 0.060 & 0.612 \\
\hline TSAT (\%) & $29.1(23.2-39.8)$ & 0.263 & 0.023 \\
\hline Homocysteine $(\mu \mathrm{mol} / \mathrm{L})$ & $30.9(26.8-38.3)$ & 0.053 & 0.668 \\
\hline $\mathrm{AOPP}(\mathrm{ng} / \mathrm{mL})$ & $336.6(249.6-389.6)$ & 0.280 & 0.049 \\
\hline NT-proBNP (pg/mL) & $3,555(1,409-12,125)$ & 0.065 & 0.581 \\
\hline hsCRP (mg/L) & $3.2(1.4-7.1)$ & 0.151 & 0.006 \\
\hline IL-6 (pg/mL) & $45.9(32.8-85.4)$ & 0.340 & 0.003 \\
\hline cTnT (ng/mL) & $0.043(0.027-0.065)$ & 0.014 & 0.907 \\
\hline $\operatorname{IMT}(\mathrm{cm})$ & $0.8(0.7-0.8)$ & -0.132 & 0.347 \\
\hline LVMI $\left(\mathrm{g} / \mathrm{m}^{2.7}\right)$ & $52.9(42.1-63.9)$ & 0.290 & 0.033 \\
\hline LVEF (\%) & $68(62.5-70)$ & -0.207 & 0.104 \\
\hline
\end{tabular}

Note: Mean (M), body mass index (BMI), erythropoietin (EPO), urea nitrogen (BUN), leukocyte count (WBC), hemoglobin ( $\mathrm{Hb})$, calcium $(\mathrm{CA})$, phosphorus $(\mathrm{P})$, serum creatinine $(\mathrm{Scr})$, total cholesterol (Tch), triglyceride (TG), high density lipoprotein cholesterol (HDL-C), low density lipoprotein cholesterol (LDL$\mathrm{C}$ ), whole parathyroid hormone (iPTH), total iron binding capacity (TIBC), unsaturated iron bonding capacity (UIBC), transferrin saturation (TSAT), advanced oxidation protein products (AOPP), cardiac troponin $\mathrm{T}$ (cTnT), amino-terminal pro-B-natriuretic peptide (NT-proBNP), high sensitivity $\mathrm{C}$ reactive protein (hsCRP), intima-media thickness (IMT), left ventricular mass index (LVMI), and left ventricular ejection fraction (LVEF). 
Table 2. Comparison of hepcidin, AOPP and IL-6 between hemodialysis patients and the control

\begin{tabular}{lccc}
\hline & Hepcidin $(\mathrm{ng} / \mathrm{mL})$ & AOPP $(\mathrm{ng} / \mathrm{mL})$ & IL-6 $(\mathrm{pg} / \mathrm{mL})$ \\
\hline Control & $84.7(49.6-179.3)$ & $78.8(51.3-139.4)$ & $33.5(22.4-47.6)$ \\
MHD group & $249.2(169.0-475.2)^{*}$ & $336.6(249.6-389.6)^{*}$ & $45.9(32.8-85.4)^{*}$ \\
\hline
\end{tabular}

Note: maintenance hemodialysis (MHD), advanced oxidation protein products (AOPP). ${ }^{*} P<0.05$ vs. control.

\section{Serum levels of hepcidin, AOPP and IL-6 in hemodialysis patients}

There were 11 males and 9 females with mean age of 60 years (range $48-71$ years) in the healthy control group. There was no significant difference in gender and age between the MHD patients and the control group $(P>0.05)$ (Table 1). Results of serum levels of hepcidin, AOPP and IL-6 in hemodialysis patients and the control are shown in Table 2. It was suggested that the levels of serum hepcidin, AOPP and IL-6 in MHD patients were significantly higher than those in the control group $(P<0.05)$.

In addition, serum ferritin levels were compared between MHD patients with CVD and MHD without CVD patients. It was suggested that the level of serum ferritin in MHD patients with CVD was significantly higher than that in non-CVD patients [335.9 (206.3-514.2) ng/mL vs. $210.5(133.6-392.3) \mathrm{ng} / \mathrm{mL}, P<0.05$ ] (data not shown).

\section{Analysis of related factors of serum hepcidin in MHD patients}

Spearman correlation analysis showed that the serum hepcidin level was positively correlated with weekly EPO dose, EPO resistance index, ferritin, TSAT, AOPP, serum hsCRP, IL-6 and LVMI. On the other hand, it was negatively correlated with serum prealbumin, TIBC, UIBC and transferrin (Table 1). In addition, linear regression analysis showed that ferritin, TIBC and IL-6 were independently correlated with serum hepcidin level (Table 3).

\section{Logistic regression analysis of risk factors for CVD}

Risk factors for CVD were measured by logistic regression analysis. It was shown that higher serum hepcidin $(>249.2 \mathrm{ng} / \mathrm{mL})$ was positively related to CVD $(\mathrm{OR}=1.32,95 \%$ CI [1.20-9.56], $P=0.043)$. Age $(\mathrm{OR}=1.03,95 \% \mathrm{CI}[1.01-1.29], P=0.049)$ and smoking $(\mathrm{OR}=2.14,95 \% \mathrm{CI}$ [1.17-14.05], $P=0.043$ ) were also associated with CVD (Table 4). The above results indicated that smoking, old age and higher serum hepcidin were independent risk factors for CVD.

Table 3. Linear regression analysis of correlation between serum hepcidin and other indexes in hemodialysis patients

\begin{tabular}{lccr}
\hline & $\begin{array}{c}\text { Non-standardized } \\
\text { regression coefficient }\end{array}$ & $\begin{array}{c}\text { Standardized } \\
\text { regression coefficient }\end{array}$ & $P$ value \\
\hline Ferritin & 0.789 & 0.708 & $<0.001$ \\
TIBC & -5.795 & -0.246 & 0.032 \\
IL-6 & 4.354 & 0.209 & 0.041 \\
\hline
\end{tabular}

Note: TIBC: Total iron binding capacity. 
Table 4. Logistic regression analysis of risk factors for CVD

\begin{tabular}{lllr}
\hline & OR & $95 \%$ CI & $P$ value \\
\hline Age (Every year of growth) & 1.03 & $1.01-1.29$ & 0.049 \\
Smoking $(Y / N)$ & 2.14 & $1.17-14.05$ & 0.031 \\
Hepcidin $(>249.2 \mathrm{ng} / \mathrm{mL})$ & 1.32 & $1.20-9.56$ & 0.043 \\
\hline
\end{tabular}

Note: cardiovascular disease (CVD), confidence interval (CI), odd ratio (OR).

\section{DISCUSSION}

Hepcidin is a polypeptide discovered in 2000 , which has anti-bacterial and anti-fungal functions. It has been confirmed that hepcidin plays a key role in the regulation of iron metabolism balance in the body $[15,16]$. At present, it is believed that the targets of hepcidin in iron metabolism are duodenal epithelial cells and monocyte macrophages. In the pathogenesis of anemia in chronic diseases, the most important point is that the increase of hepcidin level leads to the abnormal release of iron. The combination of hepcidin and its receptor membrane iron transporter blocks the release of iron from macrophages and jejunal cells, and eventually results in functional or relative iron deficiency [17-19]. The expression level of hepcidin is affected by the iron level in the body, inflammation, erythropoiesis activity, hypoxia and other factors [20]. In recent years, the relationship between hepcidin, CVD and atherosclerosis has been reported in different populations. These studies suggest that hepcidin may play an important role in atherosclerosis by capturing iron from atherosclerotic plaque macrophages and increasing oxidative stress. Furthermore, hepcidin can induce endothelial dysfunction by iron retention $[17,19,21]$. It has been shown that hepcidin is independently related to arterial stiffness in hemodialysis patients by measuring brachial ankle pulse wave velocity [22]. Weerd et al. followed up 405 patients with MHD for 3 years and found that, after correction for inflammatory factors, hepcidin is associated with fatal and non-fatal cardiovascular events [19]. Another study on menopausal women showed that the distribution of iron in the body is determined by hepcidin and the ratio of hepcidin to ferritin, rather than the total iron load of the body [23]. The above findings suggest that serum hepcidin may be a new marker and a therapeutic target for CVD. Our results demonstrate that the serum hepcidin level in MHD patients with CVD is significantly higher than that in non-CVD patients. Serum hepcidin is an independent risk factor for CVD, which is consistent with the above reported results.

Left ventricular hypertrophy $(\mathrm{LVH})$ is a significant predictor of cardiovascular morbidity and mortality in patients undergoing long-term hemodialysis (HD) therapy [12]. This study indicates that there is a positive correlation between hepcidin and LVMI. Considering the possible correlation between hepcidin and arterial stiffness, the increase of arterial stiffness can cause the left ventricular hypertrophy. In addition, it is also suggested that inflammation and other factors can lead to the increase of hepcidin and left ventricular hypertrophy. AOPP is the product of serum albumin oxidized by hypochlorite, and it is a protein crosslinked polymer containing double tyrosine. AOPP mainly reflects the degree of protein oxidation, and it is also a stable index reflecting the level of oxidative stress in the body [24]. This study also showed a positive correlation between hepcidin and AOPP, which is consistent with data reported in the literature about peritoneal dialysis patients [25].

In the present study, the levels of serum hepcidin, AOPP and IL-6 in MHD patients are significantly higher than those in the normal control group, and the level of serum hsCRP is 
significantly increased, indicating that there is widespread inflammation and oxidative stress in MHD patients. Results of this study are consistent with the previous research [26]. Additionally, there is a positive correlation between serum hepcidin and hsCRP and IL-6 in MHD patients, indicating that the increase of serum hepcidin in MHD patients is related to the state of inflammation, as reported in a previous study [27]. Some studies have shown that IL-6 can regulate the expression of the hepcidin gene by the following mechanism: IL- 6 can activate the transcription activator of ferritin by combining with signal transduction and transcription activator 3 (STAT3), thereby increasing the synthesis of hepcidin $[28,29]$. The linear regression analysis in our study showed that there is an independent correlation between hepcidin and IL-6, which supports the above conclusions. Moreover, there is a negative correlation between hepcidin and prealbumin, which may be related to the malnutrition of MHD patients caused by the inflammatory state.

The results of this study show that serum hepcidin is positively correlated with hepcidin and TSAT, whereas it is negatively correlated with transferrin, TIBC and UIBC. Linear regression analysis demonstrated that hepcidin is independently correlated with ferritin and TIBC, which verifies that hepcidin is closely related to the storage and utilization of iron, and it is a key humoral regulator of iron metabolism. The results are consistent with our previous studies on non-MHD patients $[14,30]$. We also proved that there is a positive correlation between hepcidin and weekly EPO dose and EPO resistance index, suggesting that the over-expression of hepcidin may be one cause of EPO resistance in MHD patients, and the decrease of the ferritin level may increase the sensitivity to EPO. This is consistent with findings in animal experiments and clinical research, which provided evidence that the over-expression of hepcidin can weaken the sensitivity of the body to EPO [31,32].

There are still several limitations in this study. First, the sample size is relatively small, which might affect the results. Second, there are no gold standards to identify the presence of CVD. Third, there is a lack of imaging of CVD. Finally, it is unknown whether other factors may be associated with the hemodialysis procedure in patients with and without CVD. Future studies with larger sample size are needed for establishing the roles of hepcidin in CVD development in patients under MHD, and also for evaluating their predictive values in CVD diagnosis.

\section{CONCLUSION}

In summary, iron load, inflammatory state, application of iron and EPO all affect the expression of hepcidin in MHD patients. The increase of serum hepcidin level is related to CVD. Laboratory animal models abroad have shown that inhibition of hepcidin expression can reduce the iron content of macrophages and increase the cholesterol outflow capacity, resulting in the decrease of foam cells and the reduction of atherosclerosis [33]. In the future, a cohort study with a larger sample size is needed to further verify the relationship between hepcidin and CVD under MHD.

\section{REFERENCES}

1. Parfrey PS, Foley RN. The clinical epidemiology of cardiac disease in chronic renal failure. J Am Soc Nephrol 1999; 10: 1606-15. 
2. Eckardt K-U, Gillespie IA, Kronenberg F, Richards S, Stenvinkel P, Anker SD, et al. High cardiovascular event rates occur within the first weeks of starting hemodialysis. Kidney Int 2015; 88: 1117-25.

3. Ma L, Zhao S. Risk factors for mortality in patients undergoing hemodialysis: A systematic review and metaanalysis. Int J Cardiol 2017; 238: 151-58.

4. Weiner DE, Scott TM, Giang LM, Agganis BT, Sorensen EP, Tighiouart H, et al. Cardiovascular disease and cognitive function in maintenance hemodialysis patients. Am J Kidney Dis 2011; 58: 773-81.

5. Mokou M, Lygirou V, Vlahou A, Mischak H. Proteomics in cardiovascular disease: recent progress and clinical implication and implementation. Expert Rev Proteomics 2017; 14: 117-36.

6. Haurani FI. Hepcidin and the anemia of chronic disease. Ann Clin Lab Sci 2006; 36: 3-6.

7. Hunter HN, Fulton DB, Ganz T, Vogel HJ. The solution structure of human hepcidin, a peptide hormone with antimicrobial activity that is involved in iron uptake and hereditary hemochromatosis. J Biol Chem 2002; 277: 37597-603.

8. Fraenkel PG. Understanding anemia of chronic disease. Hematology 2015; 2015: 14-8.

9. Woodman R, Ferrucci L, Guralnik J. Anemia in older adults. Curr Opin Hematol 2005; 12: 123-28.

10. D'Angelo G. Role of hepcidin in the pathophysiology and diagnosis of anemia. Blood Res 2013; 48: 10-5.

11. Young B, Zaritsky J. Hepcidin for clinicians: figure 1. Clin J Am Soc Nephrol 2009; 4: 1384-87.

12. Di Lullo L, Gorini A, Russo D, Santoboni A, Ronco C. Left ventricular hypertrophy in chronic kidney disease patients: from pathophysiology to treatment. Cardiorenal Med 2015; 5: 254-66.

13. Vadhan-Raj S, Zhou X, Bueso-Ramos CE, Patel S, Benjamin RS, Ngyuen M. Interleukin-6, hepcidin, and other biomarkers in anemia of chronic disease (ACD) and chemotherapy-Induced anemia (CIA): potential therapeutic targets. Blood 2012; 120: 2086-86.

14. Pechlaner R, Kiechl S, Mayr M, Santer P, Weger S, Haschka D, et al. Correlates of serum hepcidin levels and its association with cardiovascular disease in an elderly general population. Clin Chem Lab Med 2016; 54: 151-61.

15. Devereux RB, Alonso DR, Lutas EM, Gottlieb GJ, Campo E, Sachs I, et al. Echocardiographic assessment of left ventricular hypertrophy: Comparison to necropsy findings. Am J Cardiol 1986; 57: 450-58.

16. Lankhorst CE, Wish JB. Anemia in renal disease: diagnosis and management. Blood Rev 2010; 24 : 39-47.

17. Sullivan JL. Iron in arterial plaque: a modifiable risk factor for atherosclerosis. Biochim Biophys Acta 2009; 1790: 718-23.

18. Valenti L, Swinkels DW, Burdick L, Dongiovanni P, Tjalsma H, Motta BM, et al. Serum ferritin levels are associated with vascular damage in patients with nonalcoholic fatty liver disease. Nutr Metab Cardiovasc Dis 2011; 21: 568-75.

19. van der Weerd NC, Grooteman MPC, Bots ML, van den Dorpel MA, den Hoedt CH, Mazairac AHA, et al. Hepcidin-25 is related to cardiovascular events in chronic haemodialysis patients. Nephrol Dial Transplant 2012; 28: 3062-71.

20. Nicolas G, Chauvet C, Viatte L, Danan JL, Bigard X, Devaux I, et al. The gene encoding the iron regulatory peptide hepcidin is regulated by anemia, hypoxia, and inflammation. J Clin Invest 2002; 110: 1037-44.

21. Valenti L, Dongiovanni P, Motta BM, Swinkels DW, Bonara P, Rametta R, et al. Serum hepcidin and macrophage iron correlate with MCP-1 release and vascular damage in patients with metabolic syndrome alterations. Arterioscler Thromb Vasc Biol 2011; 31: 683-90.

22. Kuragano T, Itoh K, Shimonaka Y, Kida A, Furuta M, Kitamura R, et al. Hepcidin as well as TNF- are significant predictors of arterial stiffness in patients on maintenance hemodialysis. Nephrol Dial Transplant 2011; 26: 2663-67. 
23. Galesloot TE, Holewijn S, Kiemeney LALM, de Graaf J, Vermeulen SH, Swinkels DW. Serum hepcidin is associated with presence of plaque in postmenopausal women of a general population. Arterioscler Thromb Vasc Biol 2014; 34: 446-56.

24. Witko-Sarsat V, Friedlander M, Capeillère-Blandin C, Nguyen-Khoa T, Nguyen AT, Zingraff J, et al. Advanced oxidation protein products as a novel marker of oxidative stress in uremia. Kidney Int 1996; 49: 1304-13.

25. Ulu SM, Yuksel S, Altuntaş A, Kacar E, Ahsen A, Altug A, et al. Associations between serum hepcidin level, FGF-21 level and oxidative stress with arterial stiffness in CAPD patients. Int Urol Nephrol 2014; 46: 2409-14.

26. Xu Y, Ding XQ, Zou JZ, Liu ZH, Jiang SH, Chen YM. Serum hepcidin in haemodialysis patients: associations with iron status and microinflammation. J Int Med Res 2011; 39: 1961-67.

27. Samouilidou E, Pantelias K, Petras D, Tsirpanlis G, Bakirtzi J, Chatzivasileiou G, et al. Serum hepcidin levels are associated with serum triglycerides and interleukin- 6 concentrations in patients with end-stage renal disease. Ther Apher Dial 2014; 18: 279-83.

28. Lee P, Peng H, Gelbart T, Wang L, Beutler E. Regulation of hepcidin transcription by interleukin-1 and interleukin-6. Proc Natl Acad Sci U S A 2005; 102: 1906-10.

29. Verga Falzacappa MV, Vujic Spasic M, Kessler R, Stolte J, Hentze MW, Muckenthaler MU. STAT3 mediates hepatic hepcidin expression and its inflammatory stimulation. Blood 2006; 109: 353-58.

30. Gözdemir E, Kaygusuz I, Kafali H. Is hepcidin a new cardiovascular risk marker in polycystic ovary syndrome? Gynecol Obstet Invest 2013; 75: 196-202.

31. Rubab Z, Amin H, Abbas K, Hussain S, Ullah MI, Mohsin S. Serum hepcidin levels in patients with end-stage renal disease on hemodialysis. Saudi J Kidney Dis Transpl 2015; 26: 19-25.

32. Sasu BJ, Cooke KS, Arvedson TL, Plewa C, Ellison AR, Sheng J, et al. Antihepcidin antibody treatment modulates iron metabolism and is effective in a mouse model of inflammation-induced anemia. Blood 2010; 115: 3616-24.

33. Saeed O, Otsuka F, Polavarapu R, Karmali V, Weiss D, Davis T, et al. Pharmacological suppression of hepcidin increases macrophage cholesterol efflux and reduces foam cell formation and atherosclerosis. Arterioscler Thromb Vasc Biol 2012; 32: 299-307. 